\title{
Formulation of a departmental COVID-19 contingency plan for contact tracing and facilities management
}

\author{
ST Mak ${ }^{1,2,3}$ *, FRCSEd (Ophth), FHKAM (Ophthalmology), Kitty SC Fung ${ }^{4}$, FHKAM (Pathology), \\ Kenneth KW Li ${ }^{1,2}$, FRCOphth, FHKAM (Ophthalmology)
}

This article was published on $7 \mathrm{Apr}$ 2021 at www.hkmj.org.

\author{
${ }^{1}$ Department of Ophthalmology, United Christian Hospital, Hong Kong \\ ${ }^{2}$ Department of Ophthalmology, Tseung Kwan O Hospital, Hong Kong \\ ${ }^{3}$ Quality and Safety Office, Kowloon East Cluster, Hospital Authority, Hong Kong \\ ${ }^{4}$ Department of Pathology, United Christian Hospital, Kwun Tong, Hong Kong
}

*Corresponding author: dr.makst@gmail.com
In February 2020, the ophthalmology unit of Kowloon East Cluster, in collaboration with the infection control team, formulated this contingency plan with a main aim of facilitating the contact tracing of staff and patients to control the spread of coronavirus disease 2019 (COVID-19). ${ }^{1}$ The plan also provided guidance to our staff on facilities management.

\section{Contact tracing}

We followed the definition of contacts described by the Chief Infection Control Officer Office of the Hospital Authority of Hong Kong. Contact tracing was conducted for all staff identified as a 'close contact' or a 'probable case.' 'Close contacts' were defined as healthcare workers who had cared for patient with confirmed COVID-19 (ie, within 14 days [the incubation period of COVID-192] of symptom onset or the date of diagnosis for asymptomatic patients) without wearing appropriate personal protective equipment (PPE). Appropriate PPE was considered as surgical masks for routine patient handling in the ophthalmology setting, or full PPE including N95 respirators and face shields for aerosol-generating procedures, such those involving the use of transnasal drills or cautery. ${ }^{3}$ Staff who developed fever or respiratory symptoms after exposure to a patient with COVID-19 were managed as 'probable cases'. In case of doubt, the exposure situation was discussed with the Chief Infection Control Officer Office of the Hospital Authority or the Centre of Health Protection.

In United Christian Hospital, Hong Kong, allocation of patients to consultation rooms is managed using proprietary in-house computer software called 'UQ Web'. The system allows tracking of patient movement among the different consultation rooms, facilitating tracing of doctors, nursing staff, and allied health workers who had come into contact with patients later confirmed to have COVID-19. The system can also generate a list of patients who attended the same consultation room, allowing for contact tracing. Identified patients are informed individually and advised to report any relevant symptoms. In-patients are monitored by the hospital infection control team and out-patients are monitored by the Centre for Health Protection. The risk assessment depends on the duration, proper wearing of a surgical mask, and the closeness of contact with the patient with confirmed COVID-19 during the exposure (Fig).

In addition, the contingency plan considers facilities management. Patients with confirmed COVID-19 requiring urgent ophthalmology attendance are arranged as the final scheduled appointment of the day, to minimise service disruption, if possible. The patients are examined in a designated room with a high-efficiency particulate air filtration system capable of capturing sub-micron particulate as small as $0.1 \mu \mathrm{m} .{ }^{4}$ All staff assessing the patients wear appropriate PPE. The consultation room and all instruments are disinfected with 1:49 diluted bleach or $70 \%$ alcohol, respectively. The room is vacated for 48 hours, taking reference from the hospital's practice of operating theatre air sampling. With six air changes per hour in general hospital setting, a minimum of 69 minutes is adequate to remove $99.9 \%$ of airborne contaminants. The plan also includes contingency for backup rooms. For example, our clinic has two investigation rooms for checking visual acuity and intraocular pressure. All tests were conducted in one room, and if it had to be closed for terminal disinfection, the other room could be used.

\section{Split teams}

The majority of healthcare activities require direct patient contact, and most staff cannot work from home. In anticipation of the need for quarantine or isolation of our staff, our department implemented a split team arrangement to ensure adequate 
manpower at all times. In this arrangement, staff were allocated to teams that worked on alternate work sites or work schedules. The teams were physically segregated to minimise exposure and cross-contamination, reducing potential disruption to patient care. Because our team serves two hospitals (United Christian Hospital and Tseung Kwan O Hospital), our doctors routinely visit both hospitals, sometimes on the same day. Administrative staff also provide clerical support to the eye clinics of both hospitals. To achieve split teams, our administrative staff were split into two teams with each working in one hospital only. During the peak of the COVID-19 outbreak in February 2020, administrative staff worked from home. We also considered splitting our doctors to work at one hospital only without the need to provide service at both hospitals. This split team arrangement for doctors and allied health staff was successfully implemented in August and September 2020, and December 2020 to February 2021.

\section{Conclusion}

We have regularly updated the contingency plan to reflect the latest available scientific evidence on COVID-19. This system has been used for tracing patients three times, with approximately 40 patients successfully traced each time. We encourage fellow departments and clinics to develop their own contingency plans to prepare for the next wave of COVID-19 or other future outbreaks or epidemics.

\section{Author contributions}

Concept or design: All authors.

Acquisition of data: ST Mak, KKW Li.

Analysis or interpretation of data: ST Mak, KKW Li.

Drafting of the manuscript: ST Mak, KKW Li.

Critical revision of the manuscript for important intellectual content: All authors.

All authors had full access to the data, contributed to the study, approved the final version for publication, and take responsibility for its accuracy and integrity.

\section{Conflicts of interest}

As an editor of the journal, KKW Li was not involved in the peer review process. The other authors have no conflicts of interest to disclose.

\section{Funding/support}

This commentary received no specific grant from any funding agency in the public, commercial, or not-for-profit sectors.

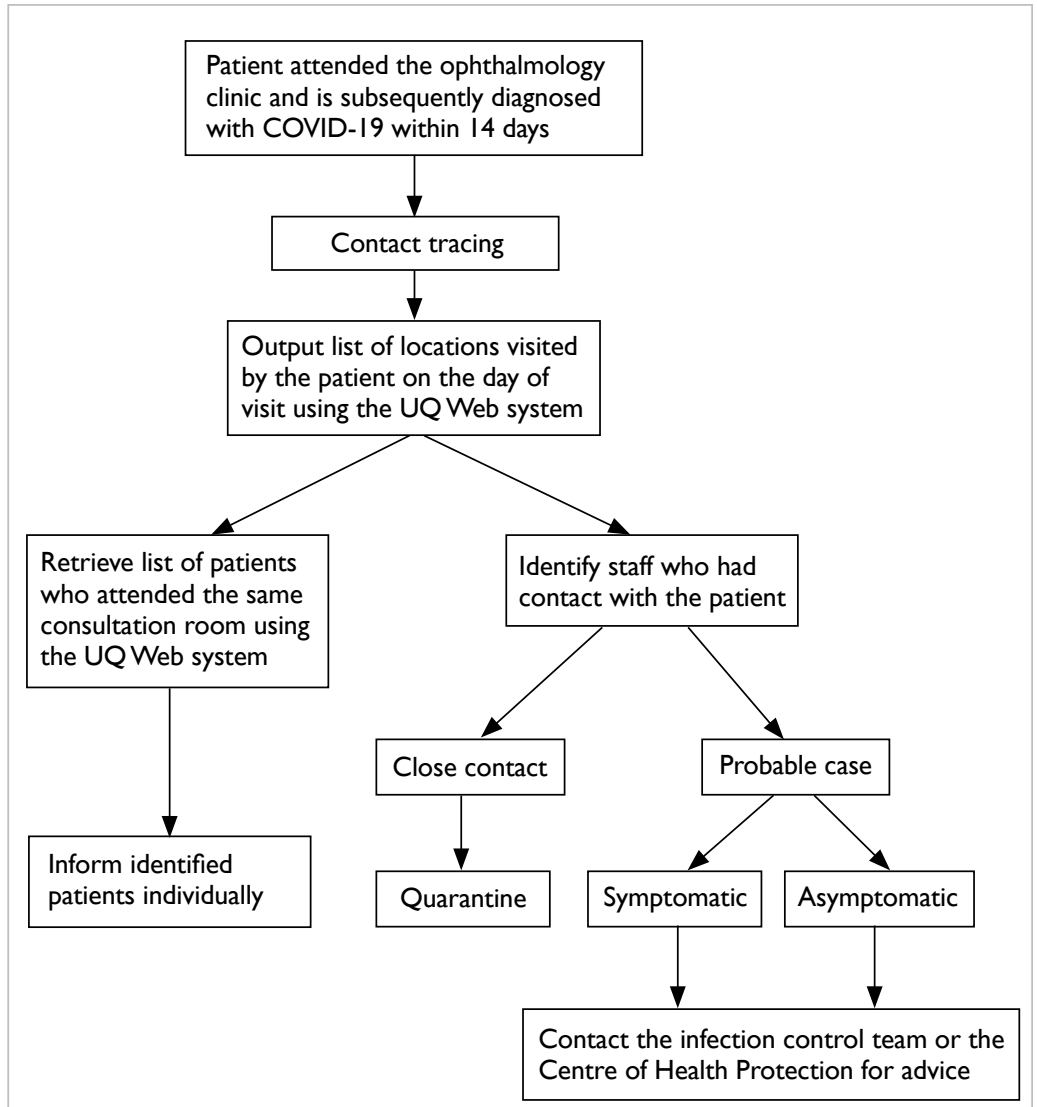

FIG. Flowchart of actions that will be carried out if a patient recently attended the ophthalmology unit is subsequently diagnosed of coronavirus disease 2019 (COVID-19)

\section{References}

1. Hellewell J, Abbott S, Gimma A, et al. Feasibility of controlling COVID-19 outbreaks by isolation of cases and contacts. Lancet Glo Health 2020;8:e488-96.

2. Linton NM, Kobayashi T, Yang Y, et al. Incubation period and other epidemiological characteristics of 2019 novel coronavirus infections with right truncation: a statistical analysis of publicly available case data. J Clin Med 2020 17;9:538.

3. Workman AD, Jafari A, Welling DB, et al. Airborne aerosol generation during endonasal procedures in the Era of COVID-19: risks and recommendations. Otolaryngol Head Neck Surg 2020 May 26. Epub ahead of print.

4. Perry JL, Agui JH, Vigayakumar R. Submicron and nanoparticulate matter removal by HEPA-rated media filters and packed beds of granular materials. NASA technical report 2016: NASA/TM-2016-218224, M-1414. Available from: https://ntrs.nasa.gov/search. jsp?R=20170005166. Accessed 2 Jul 2020. 